\title{
HOT PLASMA IN THE GALAXY
}

\author{
S. YAMAUCHI \\ Faculty of Humanities and Social Sciences, Iwate University, \\ 3-18-34 Ueda, Morioka, Iwate 020, Japan
}

\section{Introduction}

In the X-ray band, we can see weak and extended X-rays along the Galactic plane and near the Galactic Bulge region, although these regions are dominated by many point sources (e.g., Warwick et al. 1985). The Tenma satellite discovered conspicuous emission lines from selected regions near the Galactic plane (Koyama et al. 1986). These lines are identified with $\mathrm{K}$-shell line from $\mathrm{He}$-like $\mathrm{Fe}$, hence the extended emission is attributable to optically thin hot plasmas with temperatures of several $\mathrm{keV}$. The origin of the thin hot plasmas, however, have been debatable, because no class of X-ray objects shows such high temperature plasma emissions. To investigate the origin of the extended X-rays, we are currently observing the Galactic plane regions with the ASCA satellite. In this paper, we report on the ASCA results: the hard X-ray imaging and spectroscopy of the hot plasma in the Galaxy.

\section{Galactic Ridge Plasma}

At first, I present the results of the hot plasma along the Galactic plane, the Galactic Ridge emission. Figure 1 (a) shows an X-ray mosaic image in the $l \sim 28^{\circ}$ region, the direction of the Galactic arm, called the Scutum arm. Several weak discrete sources are found, but most of the X-rays are unresolved. Therefore, with the ASCA spatial resolution of about $1^{\prime}$, we confirmed that the Galactic Ridge X-rays are mainly due to diffuse hot plasmas.

Figure 1 (b) shows a typical X-ray spectrum of the Galactic Ridge emission. We see emission lines from highly ionized $\mathrm{Si}, \mathrm{S}$, and Fe. In fact, the X-ray spectra from all the observed regions are well represented by 2-temperature plasma models. The temperatures of the soft and the hard 

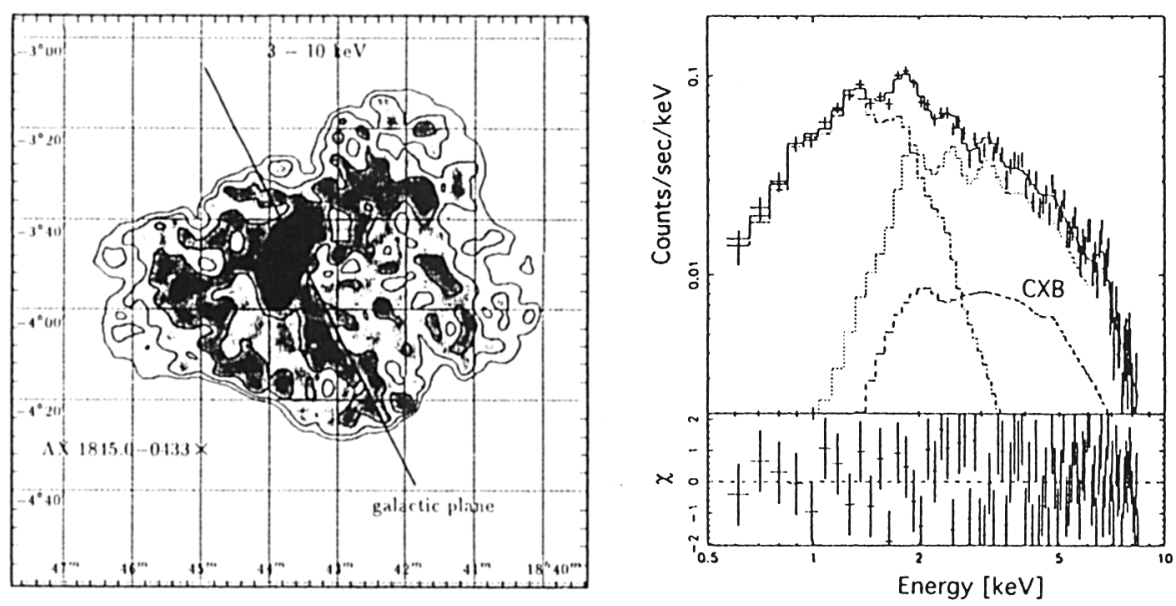

Figure 1. X-ray mosaic image of the Galactic Ridge emission in the $3-10 \mathrm{keV}$ energy band (left) and a typical X-ray spectrum (right).

components are found to be $0.6-1 \mathrm{keV}$ and $5-10 \mathrm{keV}$, respectively. The soft component can be explained by an integrated emission of supernova remnants or superbubbles, but the hard component is puzzling. Therefore, in this paper we will concentrate on the hard component, which is dominated above $3 \mathrm{keV}$.

The Galactic plane distribution of the hard component is found to be globally symmetric with respect to the Galactic Center, which agrees well with results of the $6.7 \mathrm{keV}$ iron line mapping performed with Ginga (Koyama et al. 1989; Yamauchi and Koyama 1993). Further details of the Galactic Ridge emission are presented in Kaneda et al. (1997).

\section{Galactic Center Plasma}

Since the $6.7 \mathrm{keV}$ iron line is originated from a thin hot plasma, we can know the location of the hot plasma using this line. With the Ginga satellite, we discovered an enhancement of the line flux near the Galactic Center (Koyama et al. 1989; Yamauchi et al. 1990), which means that a large amount of hot plasma is located in the Galactic Center region. The surface brightness distribution of the hot plasma is elliptical with the major and minor axes of $1.8^{\circ}$ and $1.0^{\circ}$, respectively.

In order to investigate the Galactic Center plasma in further detail, we have made the ASCA $6.7 \mathrm{keV}$ iron line map in the inner $1^{\circ} \times 1^{\circ}$ region, as is given in figure 2 . We see two diffuse plasma components near the Galactic 


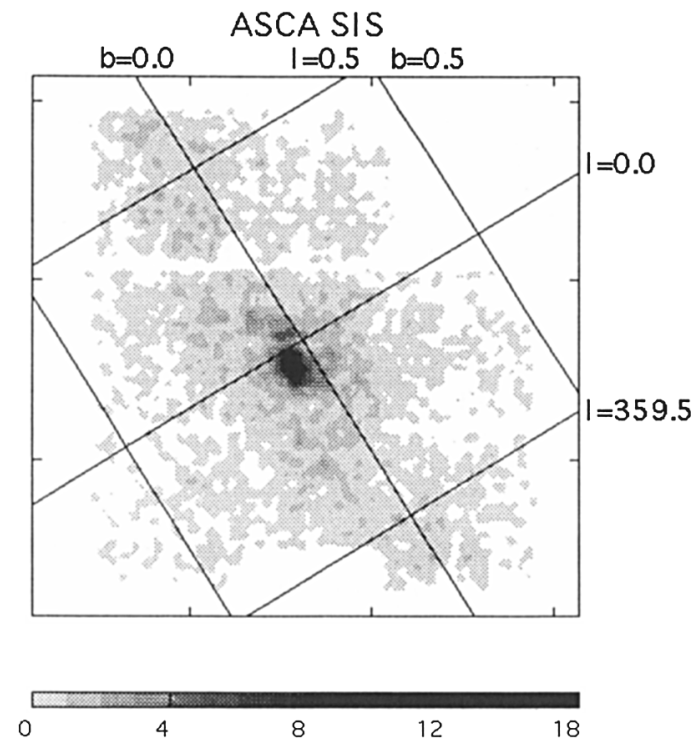

Figure 2. The $6.7 \mathrm{keV}$ iron line map in the Galactic Center region.

Center. One is the extended emission which was already discovered with Ginga. The other is newly found component, and is more compact at the Galactic Center, with the size of about $3^{\prime} \times 2^{\prime}$.

We have made X-ray spectra from 8 regions of the Galactic Center, and fitted with thin thermal models. Then we found that the intrinsic spectra are very similar with each others at the plasma temperature of about 10 $\mathrm{keV}$, while the $N_{H}$ value is variable from position to position. Therefore, we infer that the Galactic Center plasma is in the same physical conditions over the entire region.

\section{Galactic Bulge Plasma}

We also found diffuse excess $\mathrm{X}$-rays above the background level in the Galactic Bulge region. The X-ray flux decreases as the Galactic latitude increases from $|b|=0^{\circ}$ to $|b|=3^{\circ}$. However, the spectra above $3 \mathrm{keV}$ are similar to that of the Galactic Center. In fact, by model fitting, we obtained the plasma temperatures of the Galactic Bulge region to be $5-10 \mathrm{keV}$.

\section{Summary and Discussion}

We summarize physical parameters of the hot plasma in table 1 , where the dynamical time scales may indicate the maximum age of the hot plasma. We 
found that the hot plasmas with temperatures of $5-10 \mathrm{keV}$ and essentially the physical parameters, are widely distributed in the Galaxy.

TABLE 1. Physical parameters of the hot plasma in the Galaxy

\begin{tabular}{lcccc}
\hline Parameter & Ridge & Center $\left(1.8^{\circ} \times 1.0^{\circ}\right)$ & Center $\left(3^{\prime} \times 2^{\prime}\right)$ & Bulge \\
\hline Temperature $(\mathrm{keV})$ & $5-10$ & $\sim 10$ & $\sim 10$ & $5-10$ \\
Thermal energy (erg) & $\sim 10^{56}$ & $\sim 10^{54}$ & $\sim 3 \times 10^{50}$ & $\sim 10^{55}$ \\
Dynamical time scale $(\mathrm{yr})$ & $\sim 10^{5}$ & $\sim 10^{5}$ & $\sim 4 \times 10^{3}$ & $\sim 10^{6}$ \\
\hline
\end{tabular}

We discovered two hot gas components in the Galactic Center region, with the temperatures much higher than that bounded by the Galactic gravity. Together with the two different dynamical age for each plasma, we infer that the Galactic Center region has exhibited intermittent activities, which would produced the Galactic Center plasmas.

Another hint of the intermittent activities is found in the neutral iron line at $6.4 \mathrm{keV}$. The peak position of the $6.4 \mathrm{keV}$ line map agrees with the position of the molecular cloud Sgr B2 (see figure 3 in Koyama et al. 1996). The X-ray spectrum from the Sgr B2 region shows, not only the strong 6.4 $\mathrm{keV}$ line, but also a deep absorption $\mathrm{K}$-edge of iron, which is typically found in X-rays reflected by cool gas. However, in the Galactic Center region, we see no X-ray source to be reflected. This puzzle can be solved if the Galactic Center was bright 300 years ago, the light traveling time from the Galactic Center to Sgr B2. This would be the most recent activity in the central region of the Galaxy.

The author thanks Prof. K. Koyama for his helpful discussion and Dr. H. Kaneda and Mr. Y. Maeda for their kind supports to this work.

\section{References}

Kaneda, H. et al. (1997) Complex Spectra of the Galactic Ridge X-rays Observed with ASCA, Astrophys. J., in press

Koyama, K. et al. (1986) Thermal X-Ray Emission with Intense 6.7-keV Iron Line from the Galactic Ridge, $P A S J, 38,121$

Koyama, K. et al. (1989) Intense 6.7-keV Iron Line Emission from the Galactic Centre, Nature, 339, 603

Koyama, K. et al. (1996) ASCA View of Our Galactic Center: Remains of Past Activities in X-rays?, $P A S J, 48,249$

Warwick, R. S. et al. (1985) The Galactic Ridge Observed by Exosat, Nature, 317, 218

Yamauchi, S. et al. (1990) Optically Thin Hot Plasma near the Galactic Center: Mapping Observations of the $6.7 \mathrm{keV}$ Iron Line, Astrophys. J., 365, 532 